\title{
Effect of Polyethylene Interface on Space Charge Formation
}

\author{
G. Chen \\ School of Electronics and Computer Science \\ University of Southampton \\ Southampton, SO17 1BJ,UK \\ and State Key Laboratory for Electrical Insulation \\ Xian Jiaotong University, China \\ Y. Tanaka, T. Takada \\ Electronic Measurement Laboratory \\ Musashi Institute of Technology, Japan \\ and L. Zhong \\ State Key Laboratory for Electrical Insulation \\ Xian Jiaotong University, China
}

\begin{abstract}
This paper reports on an investigation into the space charge formation and decay at different material interfaces. In particular, the influence of the interface between electrode and polymer or polymer and polymer on the space charge dynamics has been studied. Planar samples were subjected to high dc electric stresses for extended periods of time and space charge measurements taken using the pulsed electroacoustic (PEA) technique. It has been found that the types of interface between electrode and polymer play a significant role in determining the charge distribution in the insulation and that the interface between polymer and polymer acts as a potential barrier to electrons while allowing positive charge carriers through easily.
\end{abstract}

Index Terms - LDPE insulation, insulation interface, space charge dynamics, PEA technique, effect of electrode materials, charge formation, charge decay, potential barrier.

\section{INTRODUCTION}

$I^{\mathrm{N}}$ $\mathrm{N}$ practice interfaces are often encountered and have become a growing area of interest due to their influence on the performance of the whole system. Cable insulation systems for example at the joints have interfaces between different materials. Unlike the case of semiconductors where the interfaces (pn junctions) are of great benefit, the interfaces in high electrically stressed materials, whether polymer/metal or polymer/polymer, can cause accumulation of space charge which can lead to unwanted stress modification. Although interfaces in joints and terminations of extruded HV cables have been identified as crucial parts, some of the mechanisms related to aging and failure are not well known. It has been reported [1] that the accumulated space charge at the interface is a more significant factor with regard to stress enhancement than in the bulk. Research into the best interfacial materi-

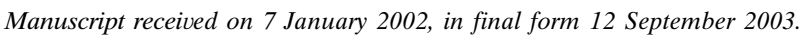

als and its binding structure is becoming increasingly important. Thermally stimulated discharge current (TSDC) technique has been used to study polymer/polymer, poly$\mathrm{mer} / \mathrm{mica}$ and polymer/oil interfaces [2-5]. Suzuoki et al [4], through their research on low-density polyethylene (LDPE)/ethylene vinyl-acetate (EVA) laminates, have proposed that the positive charges injected from the EVA side accumulate near the interface. Recently, Hozumi et al [6] have studied the charge behavior in a similar system using the pulsed electroacoustic (PEA) technique. They have found that the heterocharge is dominant at the interface. It has long been recognized that the formation of space charge at the interface whether at an electrode/polymer interface or polymer/polymer interface is more critical compared to that in the bulk because most electrical failures occur at the interfaces. It has been established that PE-based materials may have considerable concerns when used for dc power cable, particularly in the presence of voltage polarity inversions [7]. These concerns were related to the presence of space charge in 
the insulation which can increase the risk of degradation due to local stress enhancement, ionization and energy storage [8-9].

Recent research [10-12] on the effects of different electrode materials on space charge formation indicated that the electrode material has a significant effect on the charge injection and therefore on the trapping characteristics of LDPE. Three different materials, i.e. aluminum (Al), Semicon $(\mathrm{Sc})$ and gold $(\mathrm{Au})$ were used as electrodes for a single layer of LDPE [12]. From the carrier injection point of view, the results indicate that for electrons the order is as follows: $\mathrm{Sc}>\mathrm{Al}$ while $\mathrm{Au}$ injects very little; for positive charges the order is $\mathrm{Sc}>\mathrm{Al}>\mathrm{Au}$. The injection rate in the case of $\mathrm{Al}$ electrodes is positive charges $>$ electrons while with Sc electrodes the injection rate is electrons $>$ positive charges. It is also noticed the mobility of electron is much faster than that of positive charge carriers in LDPE. There are different types of semiconducting compounds available for medium and high voltage power cables. Recent investigation [13] into the effect of semiconducting screen has revealed the importance of semicon on the space charge formation in cross-linked polyethylene (XLPE).

Despite of increasing interests in the interfaces, the underlying mechanisms related to charge formation and aging are not well understood. For this reason, the present paper describes the charge formation and distribution in a system containing both electrode/polymer and polymer/polymer interfaces. Two types of electrodes are considered i.e. $\mathrm{Al}$ and $\mathrm{Sc}$ as $\mathrm{Al}$ has been widely used as electrodes in the laboratory and Sc employed in polymeric power cables. Unlike the previous research [14] where the interface is formed from different polymers, the interface in the present study is formed by laminating similar LDPE films.

\section{EXPERIMENTAL DETAILS AND TEST PROTOCOL}

LDPE was chosen initially because of its relatively simple chemical structure and its wide applications in cable insulation. Space charge formation in a material is greatly affected by the presence of impurities and additives as they can act either as ionizable centres under a high electric stress or as trapping sites. In order to reduce the influence of the impurities, additive-free low-density polyethylene (LDPE) was selected for the present study. The thickness of the sample was typically $\sim 200 \mu \mathrm{m}$ thick, consisting of two layers of $\sim 100 \mu \mathrm{m}$ thick films. Al electrodes with a diameter of $8 \mathrm{~mm}$ was evaporated on the sample under vacuum less than $2 \times 10^{-5}$ Torr. Although the influence of evaporating process on the material is not clear, it has been widely used to prepare electrodes in the laboratories. For a semicon electrode material, a thin tape was removed from the outer screen (made of Borealis Semiconducting Compound LE 0592) of a commercial

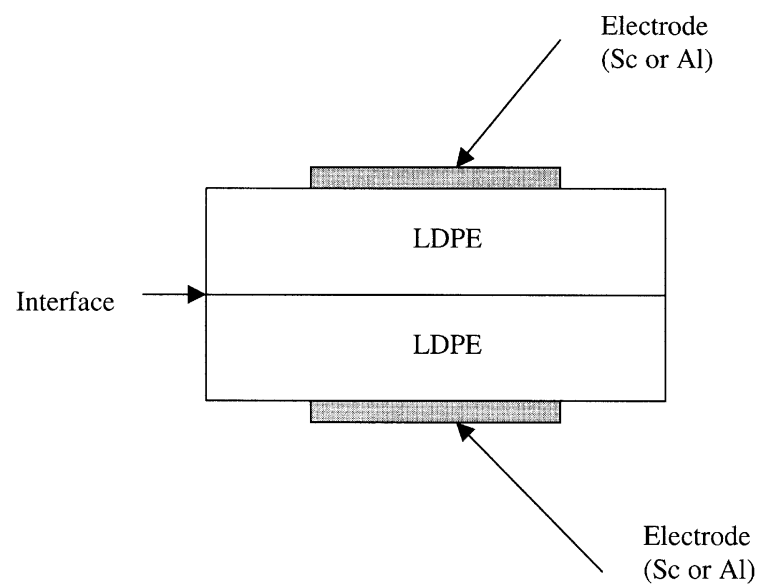

Figure 1. Schematic diagram of sample structure.

power cable; of similar size to the metallic electrode attached to the LDPE sample. The dc resistivity of Sc is less than $100 \Omega \mathrm{cm}$ at room temperature. A typical sample structure is shown in Figure 1. The interfaces in cable accessories are between rubber insulating body and cable insulation, stress-cone and epoxy body or adopter-sleeve and joint body. The interface used in the present work may be viewed as a simple representation of the interface encountered in an adaptor sleeve and joint body [15].

The electrical behavior of the interfaces is affected by several factors such as contact pressure, temperature and smoothness of the surfaces. Interfaces without microscopic cavity do not exist and some surface scratches in micron order are inevitable. In order to avoid partial discharges arising from the scratches the size of the cavities should be kept as small as possible. Care has been taken during sample preparation. The observation using the scanning probe microscopy (in the Atomic Force Mode) reveals the scratches on the surface of the LDPE films are less than $1 \mu \mathrm{m}$.

The sample was stressed at different voltage levels up to $10 \mathrm{kV}$ for a period of time at room temperature. The space charge measurements were taken using the PEA system (PEANUT ${ }^{\mathrm{TM}}$, Five Lab), which has a pulse width of $5 \mathrm{~ns}$. The sensor used was a $9 \mu \mathrm{m}$ thick $\mathrm{LiNbO}_{3}$ material that enables the system to be heated up to $90{ }^{\circ} \mathrm{C}$ although this was not utilized in the present study. A constant pressure was maintained during the measurements. The spatial resolution of the system is determined by the pulse width, acoustic speed in the material and sensor thickness and was less than $10 \mu \mathrm{m}$ which is considered adequate in the present study. The details about the PEA technique can be found in [16].

The external voltage applied to the sample was increased up to $10 \mathrm{kV}$ gradually with a voltage profile shown in Figure 2. Space charge measurements were taken at various times during the periods of both 'volts on' and 'volts off' (short-circuit condition). 


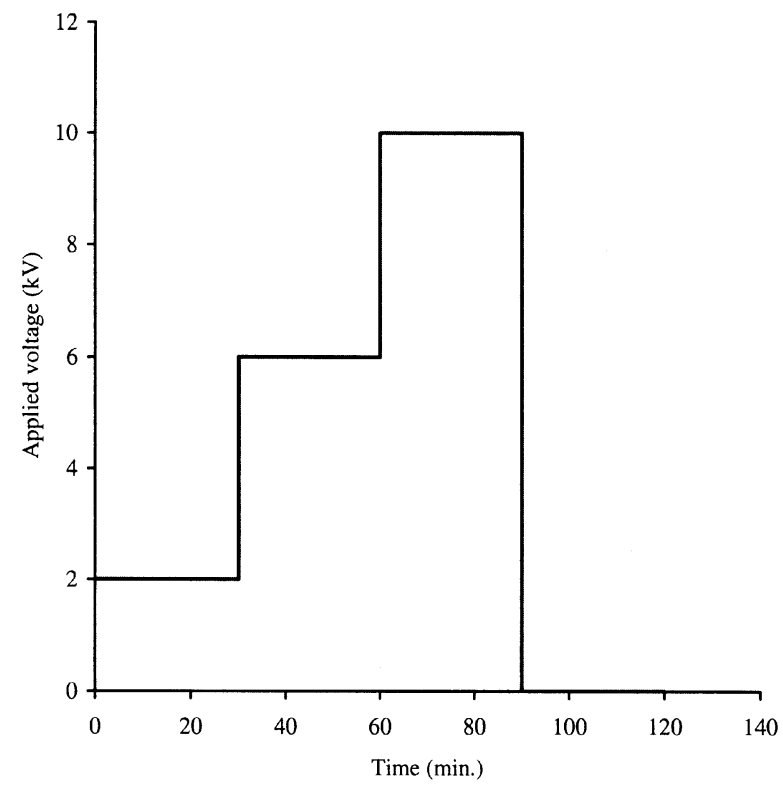

Figure 2. Voltage profile.

When an acoustic pulse travels through a material it will interact with the material. The absorption and dissipation of the acoustic energy into heat or other forms of energy are the major reasons causing attenuation. On the other hand the dispersion may be caused by the scattering of the acoustic waves (due to densely distributed inhomogeneities and frequency dependence of material constants such as elastic modulus). As a result the pulse will decrease in magnitude and broaden in width. This effect cannot be neglected if a thick sample is in question. A signal processing algorithm has been proposed [17] which recovers the signal. However, the sample used in the present study is only $\sim 200 \mu \mathrm{m}$, the effect of attenuation and dispersion is not severe, therefore no compensation has been applied in the data processing.

\section{EXPERIMENTAL RESULTS \\ 3.1 SAMPLES WITH THE SAME ELECTRODE MATERIALS}

Figures 3 and 4 show the space charge build up with 2 $\mathrm{kV}, 6 \mathrm{kV}$ and $10 \mathrm{kV}$ applied to the sample with Sc as the electrode material. The results in Figure 3 show only charge distributions at the end of each voltage application. The charge dynamics at $10 \mathrm{kV}$ is shown in Figure $4 \mathrm{a}$. The remaining charge immediately after the removal of the applied voltage and its decay are shown in Figure 4b. Since additive-free LDPE was used in this study the charge generated from ionisation of impurities in the bulk is not considered. The source for the charge measured in the sample is from electrode injection. It can be seen that charge injection takes place at a very low stress $(\sim 10$ $\mathrm{kV} / \mathrm{mm}$ ). However, its amount is small. The presence of both positive and negative charges in the sample suggests that the injection occurs at both electrodes. As expected,

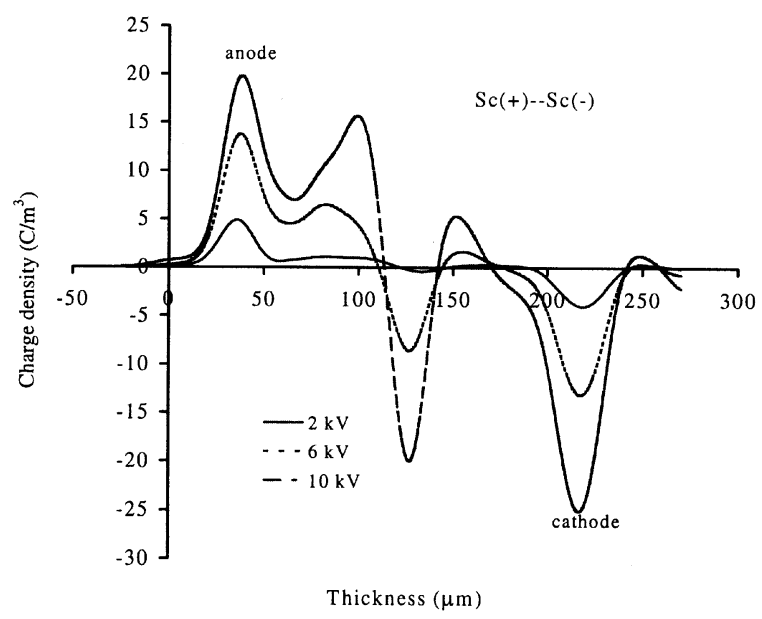

Figure 3. Charge distributions in $\mathrm{Sc}(+)-\mathrm{LDPE} / \mathrm{LDPE}-\mathrm{Sc}(-)$ with different applied voltages.

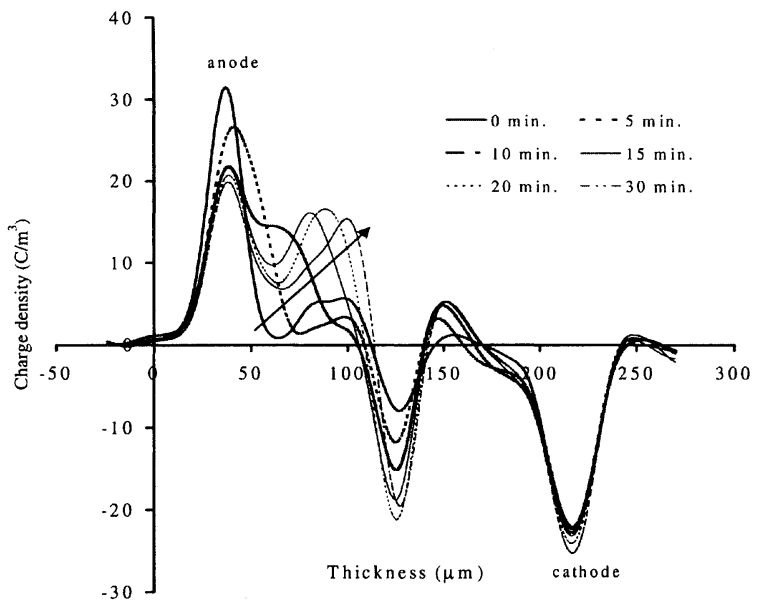

(a) Voltage on

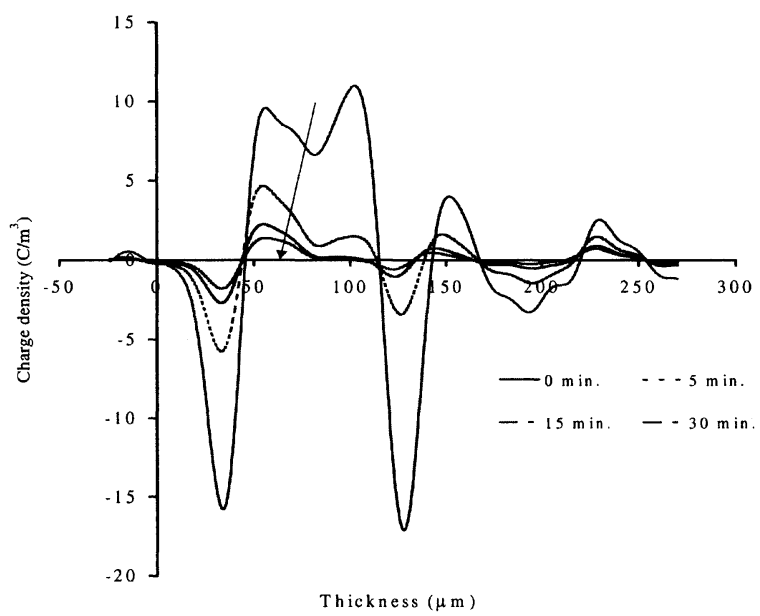

(b) Voltage off

Figure 4. Charge distributions in $\operatorname{Sc}(+)-\operatorname{LDPE} / \operatorname{LDPE}-\mathrm{Sc}(-)$ at 10 $\mathrm{kV}$. 
the amount of the injected charge increases with the applied voltage. At the end of the voltage application of 6 $\mathrm{kV}$, there is a significant amount of positive charge accumulated in the layer next to the anode with its maximum close to the polymer interface. In the layer next to the cathode, charge distribution is more complicated. There is a large amount of negative charge accumulated adjacent to the polymer interface followed by a small amount of positive charge in the middle of the layer. The broad peak due to the charge on the cathode indicates the presence of negative charge adjacent to the cathode. The above charge distribution becomes more clear when $10 \mathrm{kV}$ was applied to the sample. The positive charge injection process can clearly be seen in Figure 4a. At $\mathrm{t}=0 \mathrm{~min}$. there are positive and negative charges on both sides of the interface and these are considered as the residue charge generated during the application of $6 \mathrm{kV}$. However, at $\mathrm{t}$ $=5$ min. the peak corresponding to the charge on the anode becomes broad in width and lower in magnitude, indicating positive charge injection from the anode. The reduction in the amount of positive charge adjacent to the interface in the layer next to the anode and the increase in the amount of positive charge in the layer next to the cathode suggest that some of the positive charges are able to pass through the interface. The amount of negative charge at the interface increases with the time and these negative charges are injected from the cathode. The movement of positive charge from the anode towards the interface is also evident.

The charge distribution measured immediately after the removal of $10 \mathrm{kV}$ is in agreement with the 'volts on' observation. The charge decreases with time and the rate at which the charge decays is very fast. After 15 minutes the majority of charge in the bulk including the charge at the polymer interface diminishes through either recombination with the positive charge or conducting away from the sample.

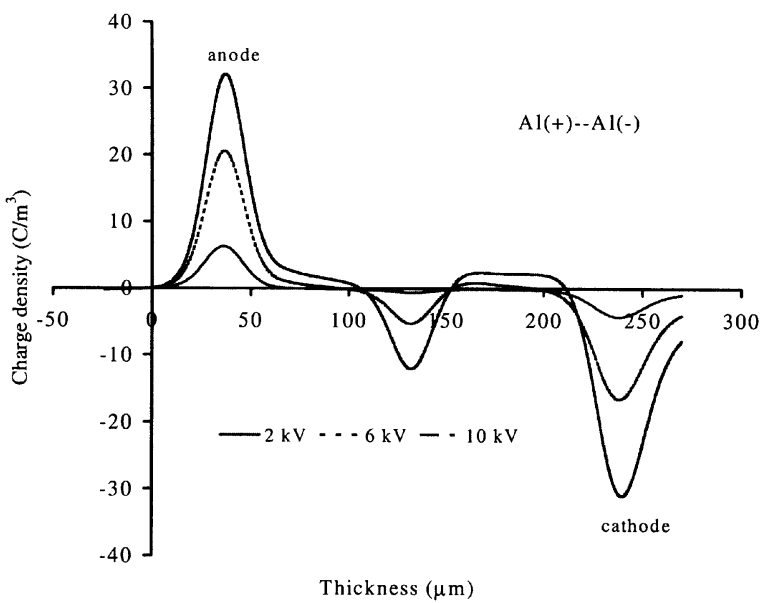

Figure 5. Charge distributions in $\mathrm{Al}(+)-\mathrm{LDPE} / \mathrm{LDPE}-\mathrm{Al}(-)$ with different applied voltages.
The charge distributions with $\mathrm{Al}$ electrodes are illustrated in Figures 5 and 6 . At $2 \mathrm{kV}$ only a small amount of negative charge can be observed at the interface. This verifies the assumption that the observed charge in the bulk is generated from charge injection from the electrodes. Otherwise if the charge were produced in the bulk via ionization there should not be any difference between Sc electrodes and $\mathrm{Al}$ electrodes at low stress. At $6 \mathrm{kV}$ more negative charges accumulate at the interface with positive charges build up on both sides of the interface. Similar behavior occurs at $10 \mathrm{kV}$. The charge dynamics at $10 \mathrm{kV}$ is not as obvious as in the sample with Sc electrodes. At $\mathrm{t}=0 \mathrm{~min}$, negative charge at the interface is dominant, this is believed to be injected from the cathode during the application of $6 \mathrm{kV}$. At $\mathrm{t}=5 \mathrm{~min}$, it is evident that the positive charge injection takes place as the peak corresponding to the charge on the anode becomes broader. The amount of negative charge at the polymer interface increases due to the injection from the cathode. However, there is a clear indication of the presence of positive

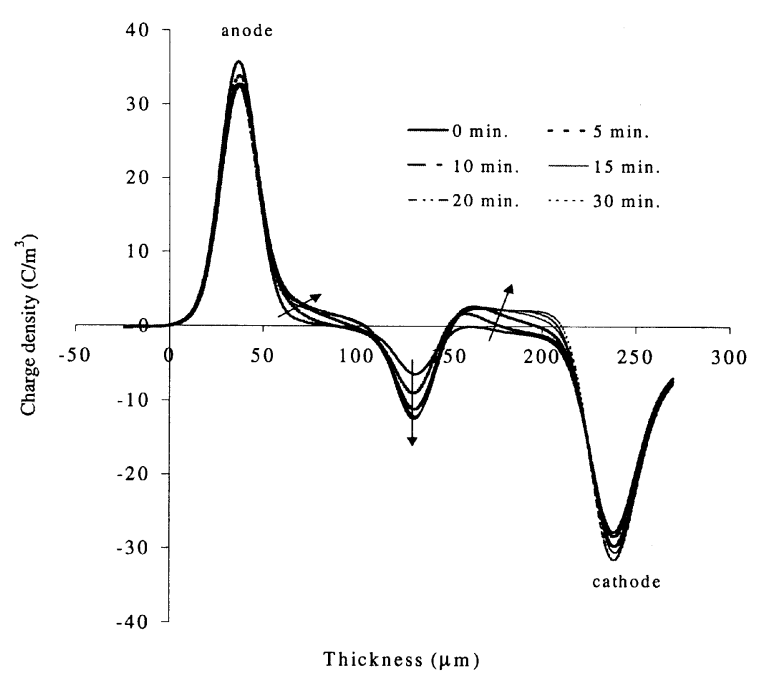

(a) Voltage on

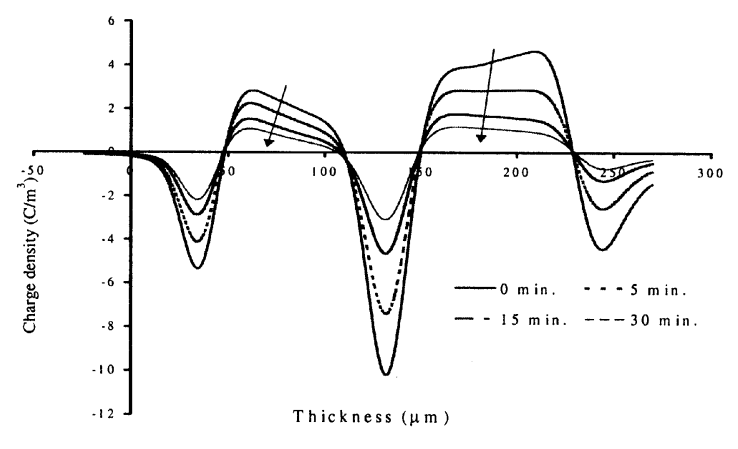

(b) Voltage off

Figure 6. Charge distributions in $\mathrm{Al}(+))-\mathrm{LDPE} / \mathrm{LDPE}-\mathrm{Al}(-)$ at $10 \mathrm{kV}$. 
charge in the layer next to the cathode. Similar to the sample with Sc electrodes, it is believed that these positive charges are part of those injected from the anode. The amount of both positive and negative charge increases with the duration of the voltage application.

Figure $6 \mathrm{~b}$ shows the space charge distribution measured after the removal of the applied voltage. Compared with Figure $4 \mathrm{~b}$, it is clear that the charge distribution is different from the sample with $\mathrm{Sc}$ electrodes. In the case of $\mathrm{Al}$ electrodes the amount of charge is generally smaller. Charge decay rate seems to be slower.

\subsection{SAMPLES WITH DIFFERENT ELECTRODE MATERIALS}

Figures 7 and 8 show the results obtained from the sample with $\mathrm{Sc}$ as the cathode and $\mathrm{Al}$ as the anode. At 2 $\mathrm{kV}$ there is a small amount of negative charge accumulated at the interface. When the applied voltage increases to $6 \mathrm{kV}$, in addition to the increase in the amount of negative charge at the polymer interface, there is a clear indication of positive charge accumulation in the layer next to the anode. Moreover, it can be seen that a small amount of positive charge is present in the layer next to the cathode with its maximum close to the polymer interface. Increase in the applied voltage leads to more charge being injected into the bulk. Figure 8a shows that the amount of charge in the bulk also increases with the duration of the voltage application. Once the applied voltage is removed the remaining charge is shown in Figure 8b. The distribution differs slightly from that when the applied voltage is on. It can be seen that negative charge appears in the layer next to the Sc electrode and positive charge in the layer next to the $\mathrm{Al}$ electrode. Again at the interface there is a large amount of negative charge. Compared with the sample with the same electrodes the amount of negative charge at the interface is lower than for Sc electrodes but higher than for $\mathrm{Al}$ electrodes.

Figures 9 and 10 illustrate the space charge formation when the polarity of the electrodes are reversed, i.e. Sc as

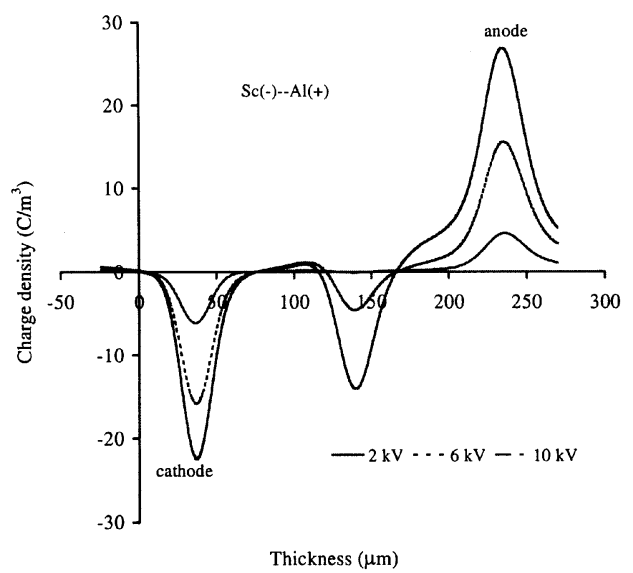

Figure 7. Charge distributions in $\mathrm{Sc}(-))-\operatorname{LDPE} / \operatorname{LDPE}-\mathrm{Al}(+)$ with different applied voltages.

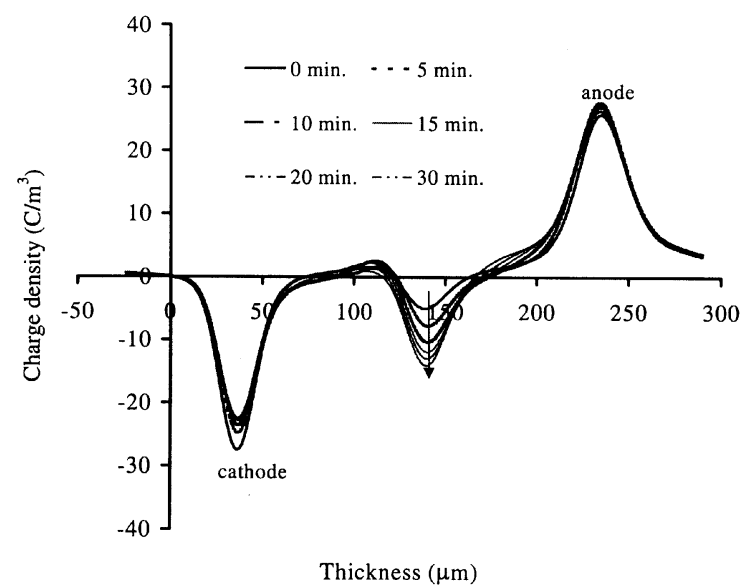

(a) Voltage on

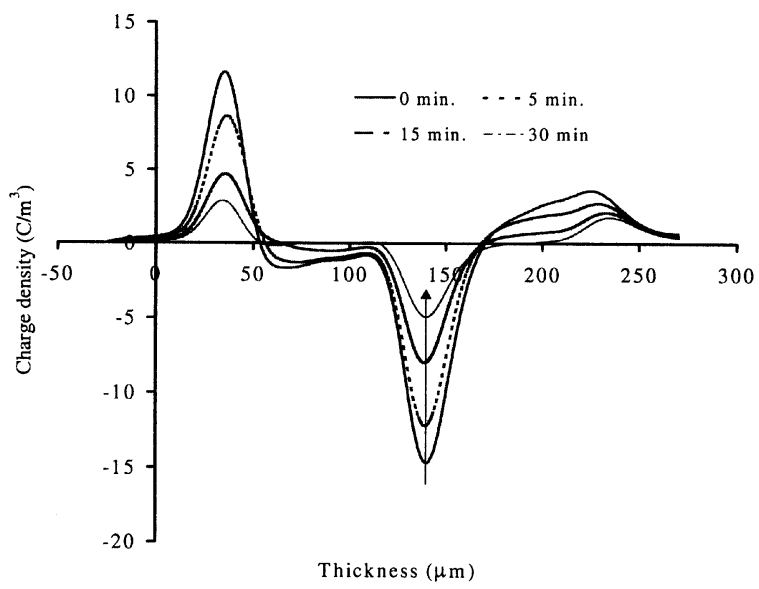

(b) Voltage off

Figure 8. Charge distributions in $\mathrm{Sc}(-)-\mathrm{LDPE} / \mathrm{LDPE}-\mathrm{Al}(+)$ at 10 $\mathrm{kV}$.

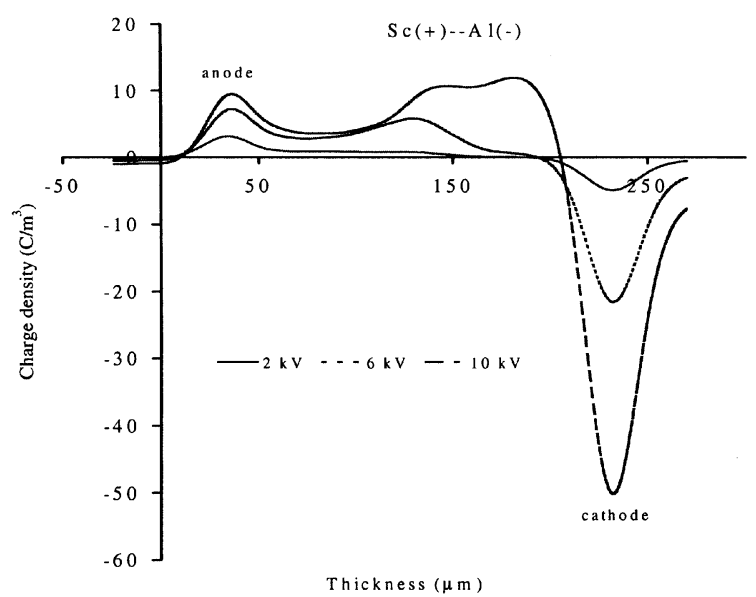

Figure 9. Charge distributions in $\mathrm{Sc}(+)-\mathrm{LDPE} / \mathrm{LDPE}-\mathrm{Al}(-)$ different applied voltages. 


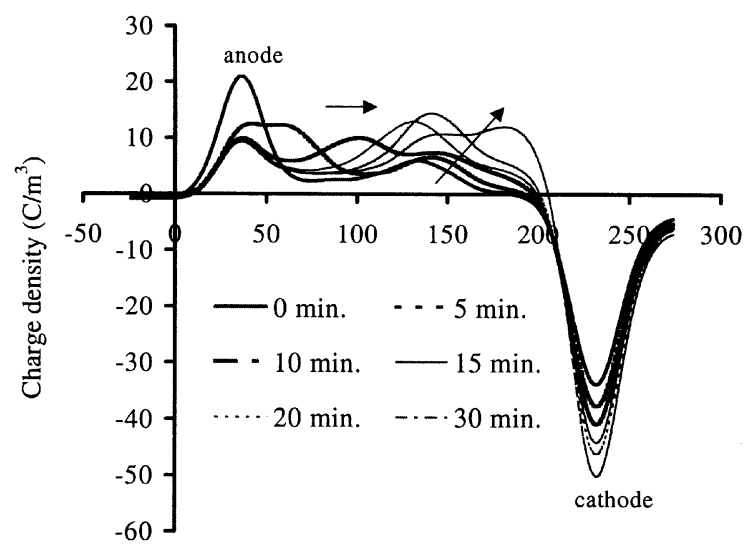

Thickness $(\mu \mathrm{m})$

(a) Voltage on

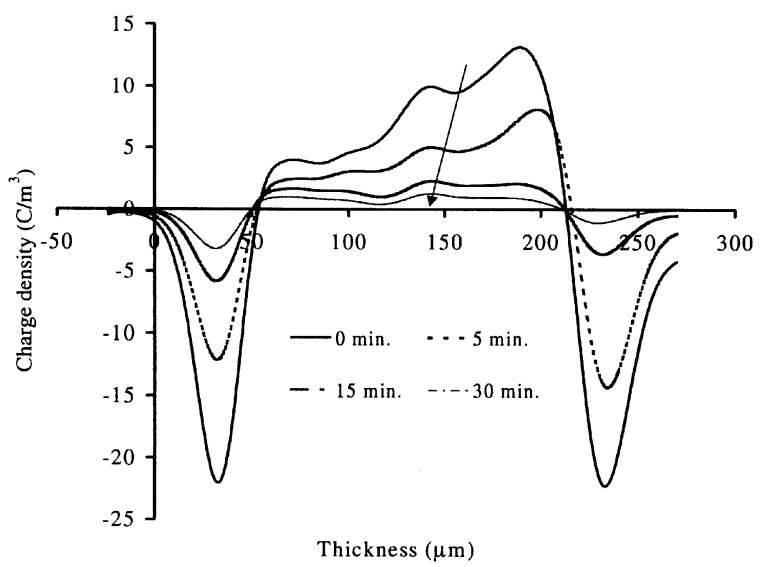

(b) Voltage off

Figure 10. Charge distributions in $\mathrm{Sc}(+)-\mathrm{LDPE} / \mathrm{LDPE}-\mathrm{Al}(-)$ at $10 \mathrm{kV}$.

the anode and $\mathrm{Al}$ as the cathode. At $2 \mathrm{kV}$ there is a small amount of positive charge in the bulk with its maximum adjacent to the anode. The amount of the positive charge increases with the applied voltage and the maximum moves from the interface with $6 \mathrm{kV}$ applied to the region adjacent to the cathode at $10 \mathrm{kV}$. Charge dynamics are clearly shown in Figure 10a; the positive charge packet is formed at the anode and moves towards the cathode. Figure $10 \mathrm{~b}$ shows the space charge distribution once the external voltage is removed. Comparing the results with those obtained previously, there are two features: only positive charge is observed across the bulk and there is no subtle change in charge distribution at the polymer interface. Charge decay in this case is fairly fast.

\section{DISCUSSIONS}

According to the electromagnetic theory [18], charge density, $\sigma$, at an interface between two dielectrics is de- termined by the following equation

$$
\sigma=\left(\varepsilon_{2}-\varepsilon_{1} \frac{\gamma_{1}}{\gamma_{1}}\right) E_{2}=\left(\varepsilon_{2} \frac{\gamma_{1}}{\gamma_{2}}-\varepsilon_{1}\right) E_{1}
$$

where $\gamma_{1}$ and $\gamma_{2}$ represent the conductivities of materials 1 and $2, \varepsilon_{1}$ and $\varepsilon_{2}$ the dielectric constants of materials 1 and 2 , respectively. This theory has been employed to describe charge formation and decay at the interfaces between different polymeric materials [19, 20], however, it experienced a difficulty in explaining quantitatively the amount of charge present at the interfaces. An attempt has been made to explain the charge formation at the interface formed between different materials such as EVA and LDPE in our earlier research [14] using the theory. It only agreed in terms of charge polarity. In the present study, as the same material is involved, therefore we have

$$
\frac{\varepsilon_{2}}{\varepsilon_{1}}=\frac{\gamma_{2}}{\gamma_{1}}
$$

i.e. the charge density at the interface formed by the same material should be zero. The observed charge at the interface clearly defies the theory. There are two main reasons for this discrepancy. Firstly, in the field range the material cannot be viewed as a linear system. It is well known that the conductivity of insulating materials is dependent on the electrical stress [21], while the permittivity of the materials is less influenced by the electric stress. Secondly, charge transportation in the material is influenced by the presence of traps and trapping characteristics. In particular, due to broken bonds and chain folds at the surfaces of the materials the traps originated from the surface states play an important role in forming charges. The charge polarity is determined by the nature of surface states.

As mentioned earlier, addition-free LDPE is used in the present study and the ionization which may form heterocharge can be discounted. Any charge measured in the bulk is considered as charge injection from the electrodes. This fact has been partially verified by the results from the samples with different electrodes shown in Figures 3 and 5. It would show the similar charge distributions if the ionization of impurities were a dominant factor in charge generation. The charge distribution clearly shows the electrode dependence and is affected by the interface.

Under the influence of the applied electric field, the injected positive charge moves towards the cathode while the injected negative charge towards the anode. Once space charges develop in the bulk of the sample due to the trapping process, these trapped charges create an electric field. The total field is the resultant field of the applied field and the space charge field.

$$
E_{\text {total }}=E_{\text {applied }}+E_{\text {space charge }}
$$

Charge injection at the electrodes is determined by the local electric field. The process may be governed by either Schottky injection which depends on the potential barrier 
height at the interface or tunnelling which strongly depends on the width of the barrier [22].

By examining the space charge distributions in the samples with different electrode combinations either with volts on or volts off, it is evident that the polymer interface acts as electron traps, but has little effect on the positive charge.

In the case of Sc electrodes, injection takes place at both electrodes. Under the influence of the electric field the injected electrons tend to move towards the anode and are blocked by the polymer interface due to the presence of electron traps. On the other hand, the injected positive charge carriers tend to move towards the cathode. Since the interface has little effect on the movement of positive charge carriers, they can move across the interface and reach the layer next to the cathode. It is believed that recombination between electrons and positive charge carriers takes place at the interface and in the layer next to the cathode due to the injected electrons distribution. This results in the charge distribution shown in Figure 3. The amount of negative charge trapped at the polymer interface reduces the field in the layer next to the cathode and enhances the field in the layer next to the anode. Consequently the injection rate increases at the anode and decreases at the cathode. The electric stress distributions in the samples with the same electrode materials are shown in Figure 11 and they are calculated by integrating the charge density

$$
E(x)=\int_{0}^{x} \frac{\rho(x)}{\varepsilon_{0} \varepsilon_{4}} d x \quad 0 \leq x \leq d
$$

From the distributions it is clear that the maximum electric field occurs at the polymer interface and the estimated field is $\sim 77 \mathrm{kV} / \mathrm{mm}$ for Sc electrodes, much higher than the applied field $(50 \mathrm{kV} / \mathrm{mm})$. This has a significant implication for power cable systems as polymer interface occurs in the joints or terminations. A high local electric

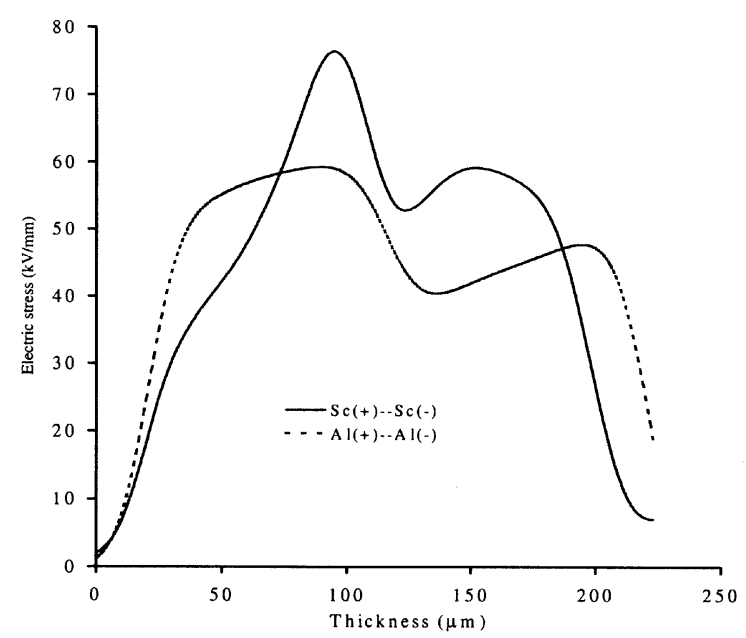

Figure 11. Electric stress distribution in samples with the same electrodes.

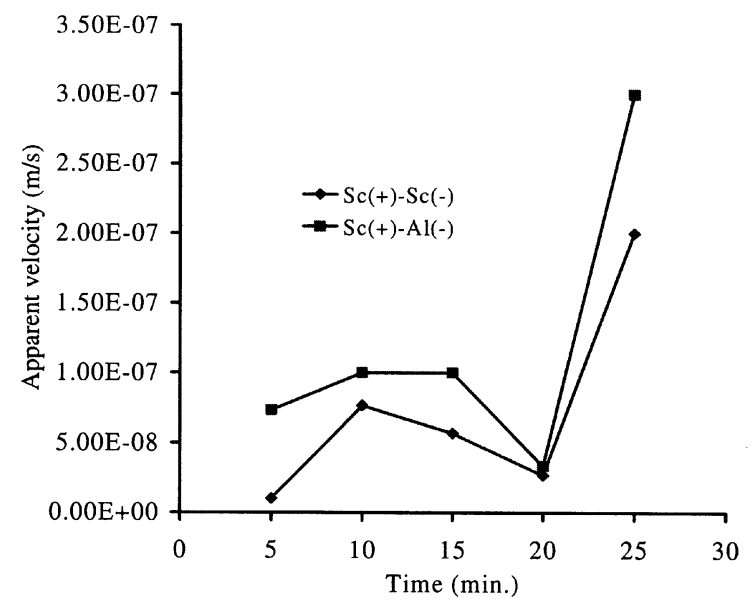

Figure 12. Apparent velocity of positive charge in the bulk at different times during the application of $10 \mathrm{kV}$.

field can lead to partial discharge activity, resulting in degradation of the material and possibly premature failure of the system.

For the $\mathrm{Al}$ electrodes, as the rate of positive charge carrier injection is greater than that of electron injection, similar to the Sc electrodes, positive charge carrier injection is enhanced by the presence of the trapped electrons at the polymer interface. Many positive charge carriers can cross the interface and distribute in the bulk of the layer next to the cathode. The recombination at the interface can also take place. Since the charge injection rate of $\mathrm{Al}$ is lower than that of Sc, the amount of charge in the bulk of the sample is less. Therefore, the electric field enhancement is not significant compared to that with Sc electrodes. The maximum electric stress is again at the interface and has a value of $60 \mathrm{kV} / \mathrm{mm}$.

In the case of Sc as the anode, the apparent velocity of positive charge carriers can be estimated by the positive peak position in the bulk of the sample as illustrated in Figure 12. It is noticed that the apparent velocity of the positive charge changes with time and the average apparent velocity in the sample with $\mathrm{Al}$ as the cathode is higher than that in the sample with Sc as the cathode, delaying the movement of the electrons. The reason is that the injected electrons from the Sc electrode can neutralise with the positive charge carriers from the anode. It has been known that the $\mathrm{Al}$ electrode injects much less electrons than the Sc electrode, consequently the recombination between electrons and positive charge carriers is limited. The movement of the charge in the bulk will also depend on the effective electric field. The velocity of charge carrier at a particular moment is affected by several factors such as the local electrical field and trapping characteristics. Some of these factors are time dependent, leading to a time dependent velocity.

Charge distributions in the samples with different electrode materials are interesting. The amount of charge and 


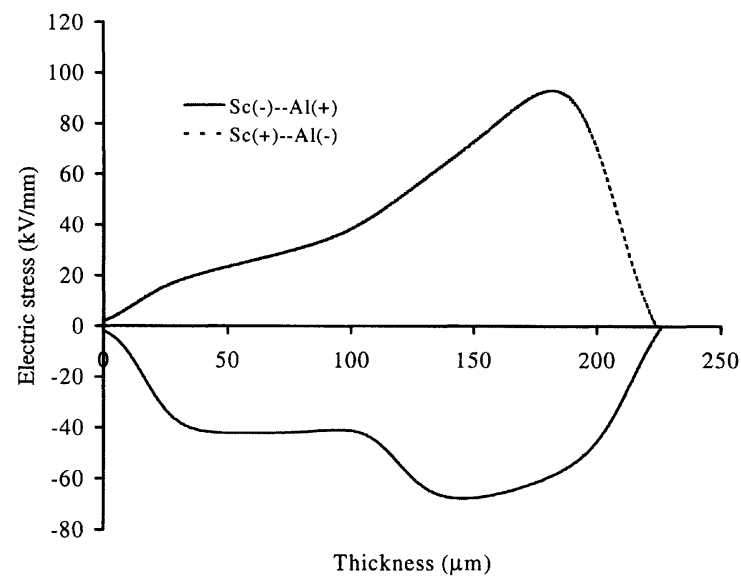

Figure 13. Electric stress distribution in samples with different electrodes.

polarity are mainly determined by the Sc electrode. The electric stress distributions after being stressed at $10 \mathrm{kV}$ for 30 minutes are shown in Figure 13. When Sc acts as the cathode and $\mathrm{Al}$ as the anode, electrons dominate with the maximum at the polymer interface. The electric field in the layer next to the $\mathrm{Al}$ electrode is enhanced, resulting in the increase in positive charge carrier injection. The positive charge carriers may be able to cross the interface but will be recombined with the large amount of electrons from the Sc electrode, hence the charge distribution as shown in Figure 8.

When Sc acts as the anode and $\mathrm{Al}$ as cathode, a large amount of positive charge carriers inject from Sc and much less electrons inject from Al. As stated earlier, the polymer interface has little effect on the movement of positive charge carrier, consequently, the positive charges distribute across the bulk of the sample with its maximum adjacent to the cathode. This kind of distribution reduces the stress in the layer next to the anode and increases the stress in the layer next to the cathode as shown in Figure 13. The increase in electric stress at the cathode enhances electron injection. However, due to lower electron injection rate of $\mathrm{Al}$ compared to higher positive charge carrier injection rate from Sc anode, any electrons injected from the cathode will be neutralised. The maximum electric stress in this case is $\sim 90 \mathrm{kV} / \mathrm{mm}$.

The apparent velocity of the positive charge movement is also illustrated in Figure 12. Compared with the sample with $\mathrm{Sc}$ as the cathode the apparent velocity of positive charge carriers in the present situation is higher. There may be two reasons for this. Firstly, the electric stress is higher and it is expected that the velocity of the charge carrier will increase with the electric stress. Secondly, the amount of electron injection from the Sc cathode is higher than that from the $\mathrm{Al}$ cathode and the neutralisation between electrons and positive charges reduces the speed of the movement of the positive charge.
On several occasions the word recombination has been used where the reduction in the amount of measured charge is observed. Care must be taken as the PEA technique only gives the resultant charge (or net charge). The reduction in charge could also be explained in terms of positive and negative charge closely located in the bulk of the sample. Further investigation is required to understand the exact mechanism causing charge reduction.

\section{CONCLUSIONS}

$\mathrm{T}_{1}$ HE effect of the interface between the two LDPE layers on space charge formation in the bulk of the sample has been reported. Sc and Al were used as electrode to form different combinations. Following conclusions may be drawn:

The interface between two layers of LDPE acts as traps for electrons but not for positive charge carriers. The charge distribution in the bulk of the sample strongly depends on the electrode materials. In the case of Sc electrodes the maximum electric field occurs at the interface. This fact needs to be taken into account when designing joints for polymeric power cable system. When different electrode materials ( $\mathrm{Sc}$ and $\mathrm{Al}$ ) are involved, the maximum electric field is likely to occur at the interface between the polymer and $\mathrm{Al}$ electrode.

\section{ACKNOWLEDGMENT}

One of the authors (GC) wishes to acknowledge the financial support from the State Key Laboratory for Electrical Insulation at Xian Jiaotong University, China.

\section{REFERENCES}

[1] Y. Li, T. Takada, H. Miyata and T. Niwa, "Observation of charge behavior in multiply low-desnity polyethylene", J. Appl. Phys., Vol. 74, pp. 2725-2730, 1993.

[2] T. Tanaka, S. Hayashi and K. Shibayama, Thermal-depolarisation-current study of composites of epoxy resin containing mica flakes, J. Appl. Phys., Vol. 48, pp. 3478-3483, 1977.

[3] T. Tanaka, S. Hayashi, S. Hirabayashi and K. Shibayama, Thermal-depolarisation-current study of composites of polystyrene containing mica flakes, J. Appl. Phys., Vol. 49, pp. 2490-2493, 1978.

[4] Y. Suzuoki, G. Cai, T. Mizutani and M. Ieda, "TSC study on interfacial phenomena in PE-EVA laminated films" Jpn. J. Appl. Phys., Vol. 21 pp. 1759-1761, 1982.

[5] T. Mizutani, M. Ieda, S. Ochiai and M. Ito, "Interfacial polarisation in silicone oil-polypropylene insulating system", J. Electrostat., Vol. 12, pp. 427-433, 1982.

[6] N. Hozumi, T. Okamoto and T. Imajo, "Space charge accumulation and decay at the interface between polyethylene and ethylene-vinyl acetate copolymer", Proc. 8th ISH, Yokohama, Japan, pp. 111-114, 1993.

[7] G. C. Montanari and D. Fabiani, "Evaluation of dc insulation performance based on space charge measurements and accelerated life tests", IEEE Trans. Dielectr. Electr. Insul., Vol. 7, pp. 322-328, 2000

[8] Y. Zhang, J. Lewiner, C. Alquie and N. Hampton, "Evidence of strong correlation between space charge buildup and breakdown in cable insulation", IEEE Trans. Dielectr. Electr. Insul., Vol. 4, pp. 778-783, 1997. 
[9] L. A. Dissado, G. Mazzanti and G. C. Montanari, "The role of trapped space charges in the electrical ageing of insulation materials", IEEE Trans. Dielectr. Electr. Insul., Vol. 4, pp. 496-506, 1997.

[10] M. A. Brown, G. Chen, A. E. Davies, L. A. Dissado and P. A. Norman, "Space charge characterisation in aged LDPE amalgamated insulation regions from underwater telecommunication systems", IEEE Trans. Dielectr. Electr. Insul., Vol. 7, pp. 346-352, 2000.

[11] Y. F. F. Ho, G. Chen, A. E. Davies, R. N. Hampton, S. G. Swingler and S. J. Sutton, "Do semicons affect space charge?", IEEE ICSD'01, Eindhoven, The Netherlands, pp. 105-108, 2001.

[12] G. Chen, T. Y. G. Tay, A. E. Davies, Y. Tanaka and T. Takada, "Electrodes and charge injection in low-density polyethylene-an experimental investigation using the pulsed electroacoustic technique", IEEE Trans. Dielectr. Electr. Insul. Vol. 8, pp. 867-873, 2002.

[13] Y. F. F. Ho, G. Chen, A. E. Davies, S. G. Swingler, S. J. Sutton and R. N. Hampton, "Effect of semiconducting screen on the space charge dynamic in XLPE and polyolefin insulation under $\mathrm{dc}$ and $50 \mathrm{~Hz}$ ac electric stresses conditions", IEEE Trans. Dielectr. Electr. Insul., Vol. 10, pp. 393-403, 2003.

[14] G. Chen, M. A. Brown, A. E. Davies, C. Rochester and I. Doble, "Investigation of space charge formation at polymer interface using the laser induced pressure pulse technique", IEEE 9th Intel. Symp on Electret, Shanghai, China, pp. 285-290, 1996.

[15] CIGRE Joint Task Force 21/15, "Interfaces in accessories for extruded HV and EHV cables", Electra, No. 203, pp. 53-59, 2002.

[16] T. Maneo, T. Futami, H. Kushibe, T. Takada and C. M. Cooke, "Measurement of spatial charge distribution in thick dielectrics using the pulsed electroacoustic method", IEEE Trans. Electr. Insul., Vol. 23, pp 433-439, 1988.

[17] A. E. Davies, G. Chen and A. Vazquez, "Space charge measurement in dispersive dielectrics", Versailles, JICABLE'99, pp. 733-738, 1999.

[18] J. C. Maxwell, A Treatise on Electricity and Magnetism, Clarendon, 1881.

[19] L Askew, "Space charge in epoxy resins measured with the pulsed electroacoustic technique", Ph.D. thesis, King's College, University of London, 1998.

[20] T. Tanaka and M. Uchiumi, "Two kinds of decay time constants for interfacial space charge in polyethylene-laminated dielectrics”, IEEE CEIDP, Austin, USA, pp. 472-475, 1999.

[21] S. Boggs, D. H. Damon, J. Hjerrild, J. T. Holboll and M.Henriksen, "Effect of insulation properties on the field grading of solid dielectric dc cables", IEEE Trans. Power Delivery, Vol. 16, pp. 456-461, 2001.

[22] K. C. Kao and W. Hwang, Electrical Transport in Solids, Oxford, Pergamon, 1981.

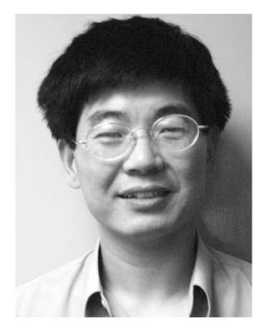

George Chen was born in China in $1961 . \mathrm{He}$ received the B.Eng. and M.Sc. degrees in Electrical Engineering from Xian Jiaotong University, China in 1983 and 1986, respectively. After he obtained the Ph.D. degree from the University of Strathclyde, UK, in 1990 he joined the University of Southampton as a Postdoctoral Research Fellow and became a Senior Research Fellow subsequently. In 1997 he was appointed as a Research Lecturer and promoted to a Reader in 2002 . He was a Visiting Scientist at Musashi Institute of Technology in Japan in 1998 and at Xian Jiaotong University in China in 1998 and 2000. He is a guest scientist in the State Key Lab of Electrical Insulation for Power Equipment, China. He was an invited speaker at the IEEE 2002 High Voltage Workshop. Over the years, he has developed a wide range of interests in HV engineering and electrical properties of materials and has published over 80 papers.

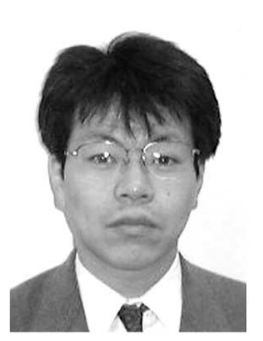

Yasuhiro Tanaka (M'99) was born in Fukuoka, Japan on 10 September 1961. He received the B.E., M.E. and Ph.D. degrees in electrical engineering from Waseda University, Japan, in 1986, 1988 and 1991, respectively. He became a Lecturer and an Associate Professor at Musashi Institute of Technology, respectively in 1992 and 1998. He was a Visiting Scientist at the University of Southampton from 1999 to 2000 . Currently, he is developing a measurement system for the space charge distribution in various solid dielectric materials at high temperatures, under ultra-high electric field or under irradiation of gammaray or electron-beam in vacuum.

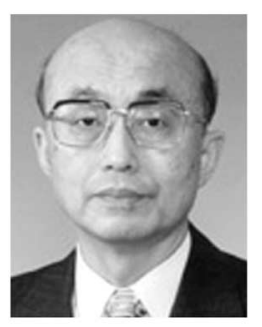

Tatsuo Takada (M'78-SM'91-F'99) was born in Yamanashi, Japan on 8 August 1939. He received the B.E. degree in electrical engineering from Muashi Institute of Technology, Japan, in 1963 and the M.E. and Ph.D. degrees from Tohoku University, Japan in 1966 and 1975 , respectively. He became a Lecturer, an Associate Professor and a Professor at Musashi Institute of Technology at Musashi Institute of Technology, respectively in 1967, 1974 and 1987. He was a Visiting Scientist at MIT (USA) from 1981 to 1983 . He received the Excellent Paper Award from the IEE of Japan in 1974, 1981 and 1990. In 1999, Dr. Takada received the Whitehead Memorial Lectureship at the IEEE CEIDP. Currently, he is involved with several research projects on the space charge effects in solid dielectric materials, surface charges on thin-film, and electric field measurements in liquid materials.

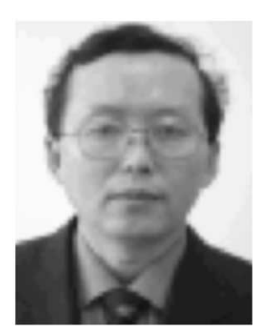

Lisheng Zhong was born in Sichuan, China, on 17 November 1961 . He graduated from Xi'an Jiaotong University and obtained the B.Sc., M.Sc. and Ph.D. degrees in 1983, 1986 and 1996, respectively. He was a Visiting Scientist at the University of Southampton from 1996 to 1998. Currently, he is an Associate Professor with the State Key Lab of Electrical Insulation for Power Equipment, Xi' an Jiaotong University, China. He is a Director of the Electrical Insulation Research Center at Xian Jiatong University. His research interests lie in the dielectric phenomena and their applications in electrical and electronic engineering and biomedicine. 